\title{
Sustained Release of Vitamin D3 Enhanced Osseointegration Capacity: An Experimental Sheep Study
}

\author{
İbrahim Ozan Mutlu ${ }^{1,2 \mathbb{D}}$, Zihni Cüneyt Karabuda ${ }^{2 \mathbb{D}}$, Volkan Arısan ${ }^{2, * \mathbb{D}}$, Melike Sessevmez ${ }^{3 \mathbb{D}}$, \\ Emine Saldamı $^{3}$ (D), Gülistan Bekel ${ }^{4}$ (D), Yusuf Altundağ ${ }^{(1 D}$, Merva Soluk Tekkeşin ${ }^{5}$ (D)

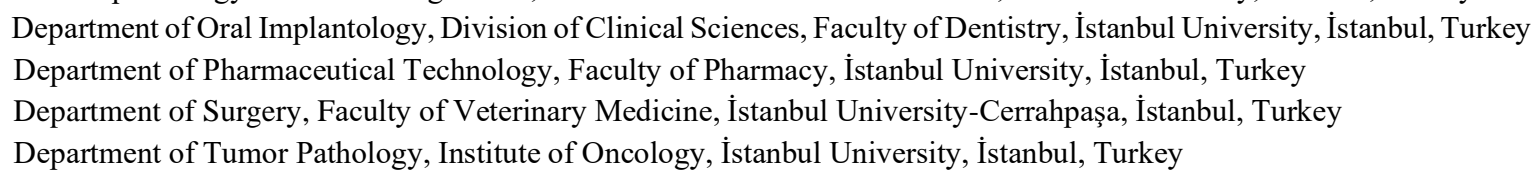

Received: 14.10.2021; Revised: 16.11.2021; Accepted: 19.11.2021; Published: 9.12.2021

\begin{abstract}
This study aimed to investigate the effect of sustained-release vitamin D loaded in poly-lactic acid (PLA) nanoparticles and applied to grafting materials around titanium (ti) dental implants.

A total of 48 implants were inserted into the standardized bone defects created in the iliac crest of 6 sheep, divided into 4 different experimental sites (empty control, autografted, xenografted, and autograft + xenografted). Vitamin D3, which was encapsulated by PLA nanoparticles, was prepared and applied in half of the defects and left to heal for 3 and 6 weeks. New bone formation (NBF\%) and bone-implant contact (BIC\%) values were evaluated by histologic and histomorphometric analyses $(P$ $<0.05)$. Nanoparticles with a yield of $80.30 \% \pm 2.14 \%$ and high encapsulation efficiency of $72.99 \% \pm$ $2.20 \%$ were achieved. In all sections, osseointegration was observed, with no signs of inflammation, necrosis, or foreign body reaction. NBF\% and BIC\% were significantly higher in vitamin D3-loaded groups $(P<0.001)$. In both healing periods, the highest NBF\% and $\mathrm{BIC} \%$ have been recorded in the autograft + xenograft groups. (For the respective third and sixth weeks: the NBF\% was $49.63 \pm 1.53$ and 74.25 \pm 0.96 , and BIC\% was $54.26 \pm 0.66$ and $82.59 \pm 2.09$, respectively; $P<0.001$ ). Sustainedrelease of vitamin D loaded into the PLA system demonstrated high biocompatibility, favorable sustained release of vitamin D3, and improved NBF\% and BIC\% around ti implants placed in boxshaped sheep iliac crest defects. Further investigations are required for their clinical applicability.
\end{abstract}

Keywords: autograft; dental implant; osseointegration; poly-lactic acid; sustained release; vitamin D3; xenograft.

(C) 2021 by the authors. This article is an open-access article distributed under the terms and conditions of the Creative Commons Attribution (CC BY) license (https://creativecommons.org/licenses/by/4.0/).

\section{Introduction}

In modern dentistry, dental implants are the most effective treatment of choice for tooth loss. Tooth loss can be caused by varied reasons, including but not limited to failed treatments, periodontal diseases, and infection, among other traumas. The triumph of modern implantology in finding solutions to such dental issues lies in prosthetically driven implant placements, which require a sufficient amount of hard and soft tissues to make the implant effective, durable, and successful [1].

In the absence of hard tissue volume, the main target in the bone augmentation process should be fulfilled with graft materials according to the patient's necessity [2,3]. In bone augmentation operations, autografts, allografts, and xenografts are frequently and commonly 
applied [4-7]. Mixing xenograft particles with autografts appears to increase the volume of newly formed bones [8]. Yet, autografts are still considered to be the golden standard when compared with other grafts $[9,10]$.

In situations where atrophic jaws do not possess the adequate alveolar bone for the prosthetically correct implant position, vertical and horizontal augmentations might be required before the placement of implants. In this regard, osseointegration and bone augmentation are complex and complicated procedures. An important patient-related factor affected the osseointegration and bone augmentation processes in bone metabolism. Sylvia Christakos et al. reported that vitamin D3 in patients was a salient factor in bone metabolism functions, and vitamin D3 deficiency directly affected bone metabolism [11].

Vitamin D3 and its metabolized 1-25 dihydroxy vitamin D3 regulate calcium, magnesium, and phosphate homeostasis levels [12]. They play a key role in calcitonin and parathyroid hormone secretion [13]. Vitamin D3 mainly targets the kidney, intestine, and bone tissues [14,15]. In bones, vitamin D3 stimulates osteoclast and osteoblast activity [16]. Moreover, vitamin D3 also functions to stimulate intestinal calcium absorption and affects the synthesis and secretion of parathyroid hormone [17]. Some of the main metabolic syndromes, such as autoimmune, cardiovascular, and infectious diseases, are caused by vitamin D3 deficiency [18].

Malnutrition and/or insufficient exposure to the sun are the major factors influencing vitamin D3 deficiency. The European Food Safety Authority recommends 600 IU/daily of vitamin D3 for all older age groups [19]. One such cause of insufficient daylight sun exposure is the modern labor conditions and the lifestyle practices followed by individuals [20]. The culmination of causes that leads to vitamin D3 deficiencies in people can lead to diseases affecting bone mineralization and metabolism, such as rickets and osteomalacia [21]. Consequently, these types of conditions impact dental implant survival and success after an osseointegration process is carried out. Regarding the use of vitamin D3 supplementation, which experts often discuss, it may affect the immune system.

Suitable transfer and efficient delivery of this critical vitamin was the main concern, and the optimal carrier has not yet been clarified. One of the most used biodegradable polymers is the poly-lactic acid (PLA)-based particles, extensively used as drug carriers to obtain a sustained release of drugs [22,23]. Appropriate carrier and encapsulating methods are used for maintaining physicochemical stability and the strength of this vitamin D3 during the bone healing period [17]. The use of vitamin D3-loaded PLA nanoparticles to explore their regeneration and osseointegration properties on box-shaped iliac bone defects in sheep has not been reported yet. Taking all these into account, the purpose of this study was to evaluate the effects of vitamin D3-loaded PLA nanoparticles on peri-implant defects, which were grafted with different biomaterials and osseointegrated in box-shaped sheep iliac crest defects.

\section{Materials and Methods}

\subsection{Animal model and experimental groups.}

This study was reviewed and approved by the Animal Experiment Ethical Committee of Mehmet Akif Ersoy Experimental Research Development and Training Center, Istanbul, Turkey (No: 2018/14). The study involved a total of six 3-year-old male sheep weighing between 50 and $70 \mathrm{~kg}$. The animals were housed in the Department Experimental Research Center of the Istanbul Mehmet Akif Ersoy Heart and Vascular Surgery Training and Research 
Hospital, Istanbul, Turkey. All experimental interventions and following care were in accordance with the required animal research guidelines and ethical codes of the center. Data obtained from a similar study [24] were referred to calculate the required sample size.

\subsection{Materials.}

The materials needed for this research included the following: PLA (RESOMER RG 203 H, Poly (D, L-lactide), acetone (EMSURE ACS, ISO, Reag. Ph Eur, Merck, Germany); Bio-Oss (Geistlich Pharma AG, Switzerland); Zinedent Dental Implant (Straumann Group Certified Partner, Ankara).

\subsection{Preparation of vitamin D3-loaded PLA nanoparticles.}

The nanoparticles were fabricated according to a previously described method employing the solvent displacement technique (4). For a brief period, $100 \mathrm{mg}$ of PLA was dissolved in $15 \mathrm{~mL}$ of acetone. Then, $10 \mathrm{mg}$ of vitamin D3 (Deva Holding Inc., Istanbul, Turkey) was added. The mixture was stirred at $250 \mathrm{rpm}$ until vitamin $\mathrm{D}_{3}$ was completely dissolved. The dispersion was added dropwise using a Pasteur pipette into the beaker containing $15 \mathrm{~mL}$ of distilled water in an ice bath, which was stirred under a magnetic stirrer at $300 \mathrm{rpm}$ for $1 \mathrm{~h}$. After this process, the organic solvent of the dispersion was evaporated (Rotary Evaporator, Heidolph) at $30^{\circ} \mathrm{C}$.

The nanoparticles loaded with vitamin D3 were obtained after the nanoparticle dispersion was centrifuged at $9500 \mathrm{rpm}$ for $1 \mathrm{~h}$ at $4{ }^{\circ} \mathrm{C}$. Subsequently, it was lyophilized (VirTis AdVantage Plus, Benchtop Freeze Dryer, SP Scientific, PA, USA) for $72 \mathrm{~h}$ at $-40^{\circ} \mathrm{C}$. After the completion of the lyophilization step, the dried nanoparticles were weighed. The production yield of vitamin D3-loaded nanoparticles was calculated using the following equation $(n=3)$ :

$$
\text { Production yield }(\%)=\frac{\text { weigh of vitamin D3 loaded nanoparticles }}{\text { Total solids }(\text { PLA }+ \text { vitamin D3 })}
$$

\subsection{Characterization of nanoparticles.}

The nanoparticles' particle size and distribution measurement (polydispersity index, PDI) were measured using photon correlation spectroscopy (Nano-ZS Malvern Instruments Ltd., UK). Additionally, the zeta potential of nanoparticles was assessed using the electrophoretic light-scattering technique (Nano-ZS Malvern Instruments Ltd.). Finally, the morphological evaluation of nanoparticles was conducted using scanning electron microscopy (SEM; FEI QuantaTM FEG 450, Oregon, USA).

\subsection{Encapsulation capacity of vitamin D3-loaded nanoparticles.}

Nanoparticles $(5 \mathrm{mg}$ ) were added into an organic solvent mixture with $0.2 \mathrm{~mL}$ of acetonitrile. Methanol $(0.8 \mathrm{~mL})$ and the mixture were vortexed for $10 \mathrm{~min}$. The mixture was then centrifuged for $5 \mathrm{~min}$ at 15,500 rpm. In addition, the supernatant part was separated and filtered using a $0.45-\mu \mathrm{m}$ filter for the further analysis of the amount of vitamin D3 via highpressure liquid chromatography (HPLC; Prominence-I LC-2030/2030C HPLC, Shimadzu, Kyoto, Japan) [25]. 
As the mobile phase, the mixture of acetonitrile:methanol $(55: 45 \mathrm{v}-/-\mathrm{v})$ was used. The flow rate was set at $1 \mathrm{~mL} / \mathrm{min}$, with an injection volume of $20 \mu \mathrm{L}$. The column temperature was set at $40^{\circ} \mathrm{C}$. The separation was conducted on a C18 column with a particle size of $5 \mu \mathrm{m}$ (Intersil ODS-3 C18, $250 \times 4.6 \mathrm{~mm}^{2}$ ).

The encapsulation efficiency (EE) was determined using the following equation $(n=$ 3):

$$
E E=\frac{\text { Actual amount of vitamin D3 in the particles }}{\text { Theoretical amount of vitamin D3 in the nanoparticles }} \times 100
$$

\subsection{In Vitro release test of vitamin D3-loaded nanoparticles.}

In this test, $15 \mathrm{mg}$ of vitamin D3-loaded nanoparticles were placed in 2-mL Eppendorf tubes; $1 \mathrm{~mL}$ of $\mathrm{pH} 7.4$ phosphate-buffered saline was added. The tubes were shaken at a speed of $150 \mathrm{rpm}$ in an orbital shaker at $37^{\circ} \mathrm{C}$ (Forma Orbital Shaker, Thermo, Massachusetts, USA). At predetermined time points $(2 \mathrm{~h}, 4 \mathrm{~h}, 6 \mathrm{~h}, 24 \mathrm{~h}, 2 \mathrm{~d}, 4 \mathrm{~d}, 7 \mathrm{~d}, 2 \mathrm{w}, 3 \mathrm{w}, 4 \mathrm{w}, 5 \mathrm{w}$, and $6 \mathrm{w})$, $0.9 \mathrm{~mL}$ of samples were taken while the total volume was preserved through replacement with fresh medium. The amount of vitamin D3 in the sample was determined through an HPLC analysis.

\subsection{Surgical procedures.}

The animals involved in the study were sedated with xylazine $[0.1 \mathrm{mg} / \mathrm{kg}$ (i.m.); Rompun, Bayer, Switzerland], and their induction was performed with Ketalar [3 mg/kg (i.v.), ketamine $\mathrm{HCl}$, Pfizer, Istanbul, Turkey]. General anesthesia was induced using sevoflurane (Eczacibasi-Baxter, Istanbul, Turkey) through intubation and maintained throughout the duration of the operation.

The animal was placed in a lateral couchant position. The medial part of the animal's bilateral ilium and acetabulum was shaved and disinfected with povidone-iodine. The ilium of the animal was accessed by opening a longitudinal incision of $25-30 \mathrm{~cm}$ at the mid-pelvis, from the ala-osis ilium to the trochanter major. After separating the skin and subcutaneous tissues, the fascia lata was incised. Subsequently, a blunt dissection of the gluteal muscle was performed. This exposed the pelvis, and the periosteum of the animal was dissected. From acetabulum to the ilium wing, four standardized box-shaped defects were created $(5 \times 10 \times 5$ $\mathrm{mm}^{3}$ ) with the help of a diamond disk (MicroSaw, Dentsply, MI, USA) on each ilium through low rotational speed $(\max 800 \mathrm{rpm})$ and continuous external saline irrigation. Care was taken to ensure the uniformity and standardization of the defect shapes and dimensions via the use of a periodontal probe. The box-shaped defects were classified as follows: Nongrafted (empty control); Autografted; Xenografted; Autografted and xenografted (mixed).

Autograft particles were obtained from the defect areas that were surgically prepared using a diamond disk (MicroSaw, Dentsply). Selected xenograft particles were deproteinized bovine bone mineral Bio-Oss. After the preparation of the defect areas, sustained-release vitamin D3-loaded PLA nanoparticles were applied on the right-wing of each ilium. On the left-wing, PLA nanoparticles that did not include vitamin D3 were applied. Ensuing this, dental implants (Zinedent, Straumann Group, İstanbul) were installed in each site. Vitamin D3-loaded PLA nanoparticles equivalent to $2 \mathrm{mg}$ of vitamin D3 were applied on box-shaped iliac bone 
defects in sheep. As the last step in the procedure, augmentation processes with signified grafts were conducted (Figure 1).

The animals were randomly divided into two groups: with or without sustained-release vitamin D3-loaded PLA nanoparticles. After completing the biomaterial applications, the periosteum was closed using No. 0 poliglecaprone suture (Vetsuture PGC, Paris, France). The primary wound closure was undertaken and achieved with No. 0 vicryl suture (Ethicon Vicryl, Kalimed Medical, Ankara, Turkey).
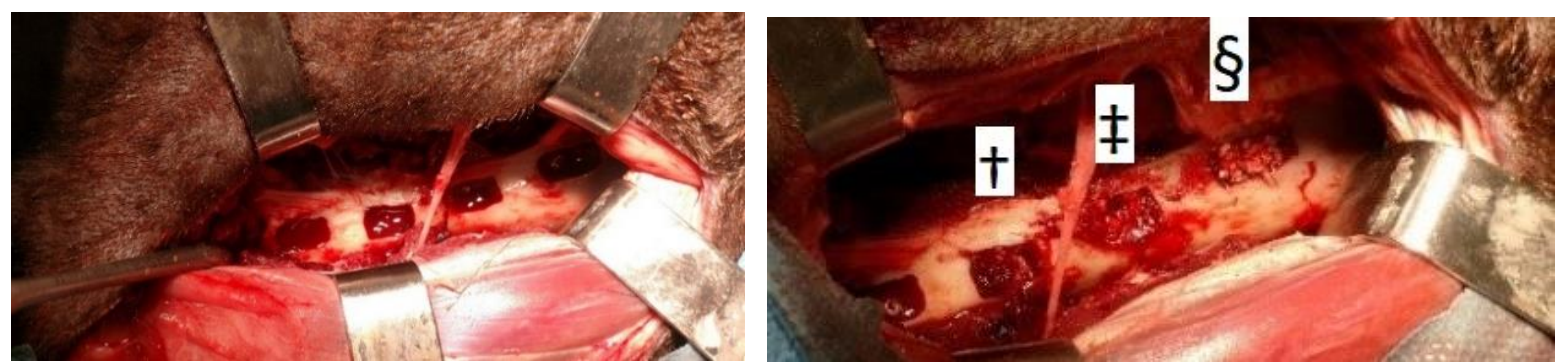

Figure 1. (a) Preparation of the box-type defects on the sheep's ilium. (b) Biomaterial applications in the two groups according to the previously determined order, from acetabulum to the ilium wing; empty control defect, defect filled with ${ }^{\dagger}$ autogenous graft, ${ }^{\star}$ xenograft, and ${ }^{\S}$ autogenous \& xenograft combination.

\subsection{Sacrification.}

Three sheep were left to heal for 3 weeks (early-term healing), and three were left to heal for 6 weeks (late-term healing). Upon completion of these periods, the animals were sacrificed following the principles of the Islamic sacrifice ritual, as requested by the Ethical Committee [26]. Iliums were removed and fixed in $10 \%$ neutral-buffered formalin solution ( $\mathrm{pH}$ 7.0) at room temperature for 2 days for the histologic and histomorphometric analyses.

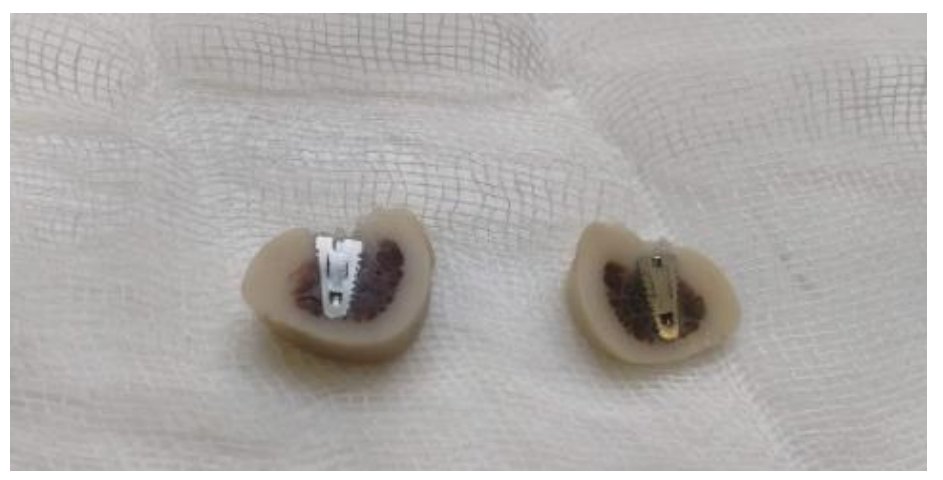

Figure 2. Longitudinal section of implant and ilium wing.

\subsection{Histological analysis.}

A bone block including four defect sites was trimmed into the blocks incorporating the surrounding bone. The blocks were dehydrated in ethanol by increasing the alcohol level $(60 \%$, $80 \%, 96 \%$, and $100 \%$ ) every $24 \mathrm{~h}$. Then, all samples were infiltrated in methyl methacrylate resin (Technovit 7200 VLC; Heraeus Kulzer GmbH \& Co. KG, Wertheim, Germany) and alcohol with increasing resin percentages $(30 \%, 50 \%, 70 \%$, and $100 \%)$ under a vacuum. Longitudinal pieces from each implant were obtained using a dedicated non decalcified histologic slicing system (Exact 300 CL; Exakt Apparatebau, Norderstedt, Germany); (Figure 2). Ground sections of $300 \mu \mathrm{m}$ were prepared, thinned to $40 \mu \mathrm{m}$, and stained with toluidine blue. 
2.10. Histomorphometric analysis.

All sections were examined using a stereomicroscope (Olympus BX60, Tokyo, Japan) and a color video camera (Olympus DP 25; Olympus Optical Co. Ltd., Tokyo, Japan). A dedicated image analysis software (Olympus Image Analysis System; Olympus Soft Imaging Solutions GmbH, Münster, Germany) was used for the histomorphometric analysis.

The whole surface of each defect site was captured in four continuous and consecutive microscopic fields. The bone-implant contact (BIC) values were determined by measuring the length of the attached bone-implant surface (osseointegrated surface) and dividing that by the whole surface perimeter at X100 magnification. In addition, the new bone formation (NBF) and soft tissue areas were measured at the interface of the bone implant. All the measurements were taken and recorded by two independent examiners experienced in the hard tissue and graft histomorphometry. The mean value from the two examiners was recorded as the final measurement.

\subsection{Statistical analysis.}

A commercial software package (NCSS-Number Cruncher Statistical System, UT, USA) was used for the statistical analysis. Descriptive statistics consisting of the mean, standard deviation (SD), median, interquartile range, range (minimum-maximum), and 95\% confidence interval were calculated for NBF and BIC parameters. Normality was not assumed. The Kruskal-Wallis test determined significant differences between the four experimental defects and two-time intervals. The Spearman correlation test was used to evaluate the relationship between the quantifiable variables of NBF and BIC. A $P$ value $<0.05$ was considered statistically significant.

\section{Results and Discussion}

\subsection{Preparation and characterization of nanoparticles.}

Vitamin D3-loaded nanoparticles were prepared via the solvent displacement method. Using this particle preparation method, the mean particle size and PDI of the blank nanoparticles and vitamin D3-loaded nanoparticles were determined $(153.34 \pm 0.95 \mathrm{~nm}$ and 0.081 , and $146.82 \pm 1.36 \mathrm{~nm}$ and 0.130 , respectively (Table 1; Figure 3). However, the high production yield and EE of vitamin D3-loaded nanoparticles were 80.30 \pm 2.14 and $72.99 \pm$ 2.20, respectively (Table 2). The particle morphology of vitamin D3-loaded nanoparticles was examined using an SEM. The nanoparticles displayed a spherical shape with a smooth surface (Figure 4).

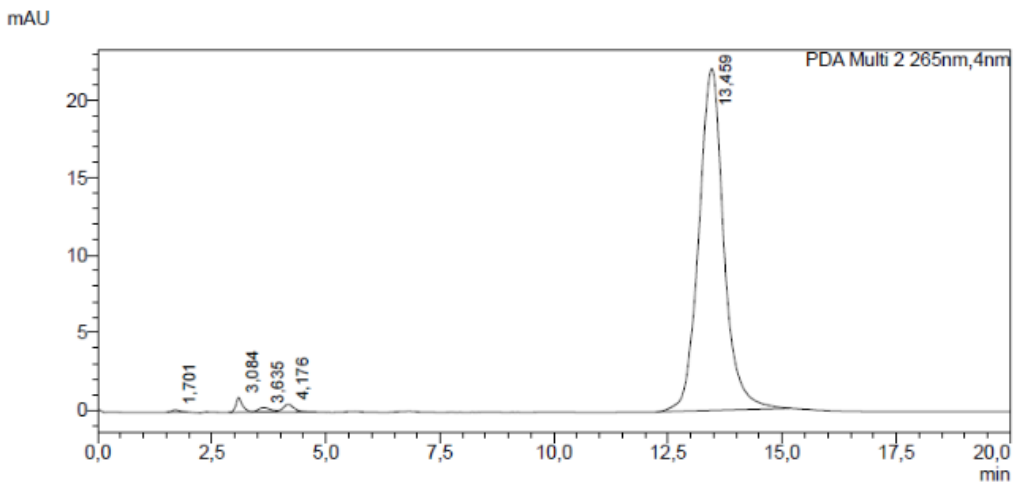

Figure 3. Chromatogram of vitamin D3 (retention time $=13.459 \mathrm{~min}$ ). 
3.2. Particle sizes and zeta potential values of nanoparticles.

Table 1. Particle Size, Polydispersity Index, and Zeta Potential of Vitamin D3 loaded PLA nanoparticles (n=3)

(a vitamin D3 loaded PLA nanoparticles, b blank PLA nanoparticles).

\begin{tabular}{c|c|c|c} 
Formulation & Particle size $(\mathbf{n m} \pm \mathbf{S D})$ & Polydispersity index $(\mathbf{P D I} \pm \mathbf{S D})$ & Zeta potential $(\mathbf{m V} \pm \mathbf{S D})$ \\
\hline A & $146.82 \pm 1.36$ & 0.103 & $-31.8 \pm 2.5$ \\
\hline B & $153.34 \pm 0.95$ & 0.081 & $-15.4 \pm 1.2$
\end{tabular}

\subsection{Yield of nanoparticles.}

Table 2. Production yield and EE of vitamin D3-loaded PLA nanoparticles $(n=3)$.

\begin{tabular}{c|c} 
Production yield $(\% \pm$ SD) & Encapsulation efficiency $(\% \pm$ SD $)$ \\
\hline $80.30 \pm 2.14$ & $72.99 \pm 2.20$
\end{tabular}

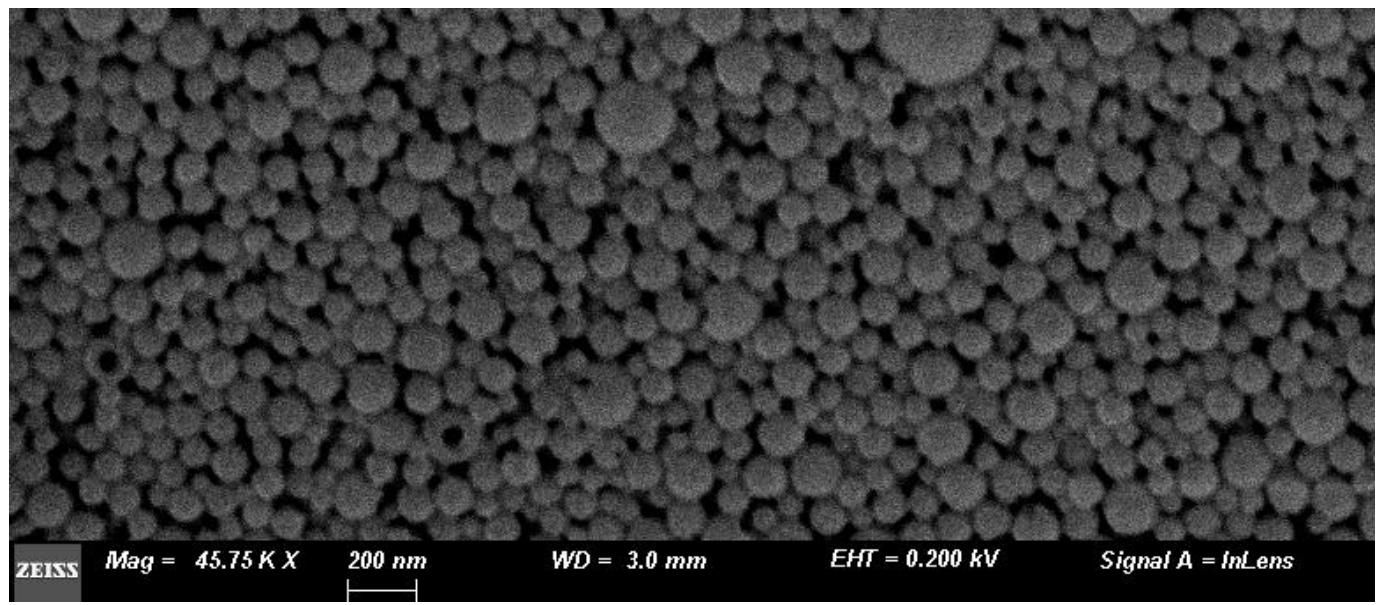

Figure 4. Scanning electron microphotograph of vitamin D3-loaded PLA nanoparticles (original magnification $\times 200)$.

\subsection{In vitro drug release.}

Sustained release and maintaining activity of vitamin D3 was expected from PLA nanoparticles. In vitro cumulative vitamin D3 release from PLA nanoparticles is shown in Figure 5. Two phases of vitamin D3 release were monitored in the drug release profile. The initial burst release of vitamin D3 was seen within the first $2 \mathrm{~h}$ (32\% of the drug was released), and then a prolonged slow-release rate of vitamin D3 was obtained $(72 \%$ of the drug was released after 9 weeks).

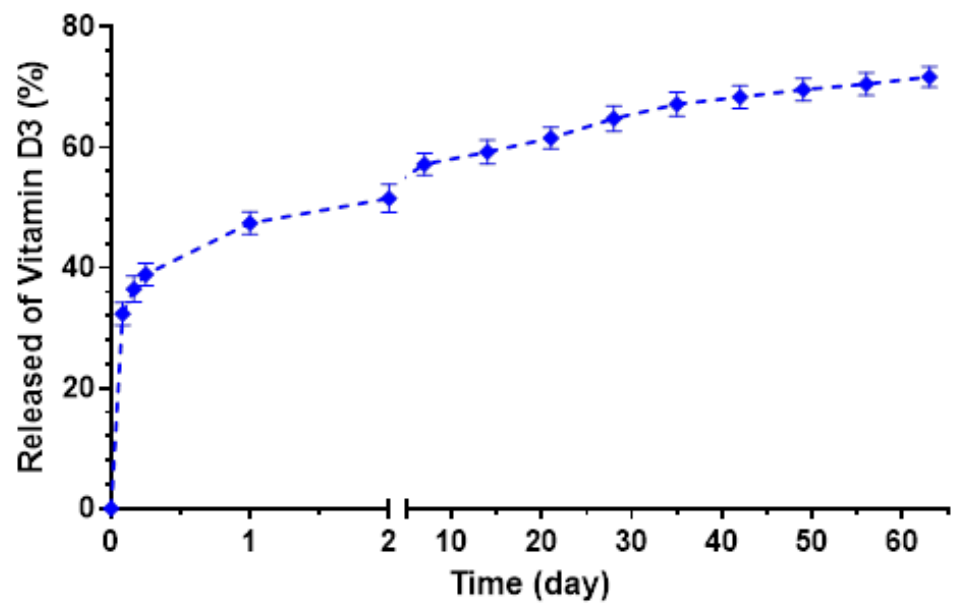

Figure 5. In vitro cumulative drug release from vitamin D3-loaded PLA nanoparticles. 


\subsection{Histological observations.}

In all implant sections, osseointegration was observed, and no indications of inflammation, necrosis, or foreign body reaction were noted. A tartrate-resistant acid phosphatase staining was unnecessary due to the absence of inflammatory cells in the histologic sections. An active osteoid deposition was discernible around the implants. However, some gaps were detected in the bone-implant interface of the implants in the vitamin D3- group versus the vitamin D3+ group. Primary osteons were more prominent in the vitamin D3+ group, while the healing of the surrounding bone appeared to be delayed among the vitamin D3group.

Active remodeling of the osseointegrated bone interface with a clear demarcation line between the host and the new bone was observed around the groups. An organized bone matrix with the primary osteons was visible compared with autogenous and xenograft around the titanium implants. The deposition of lamellar bone was observable (Figure 6).

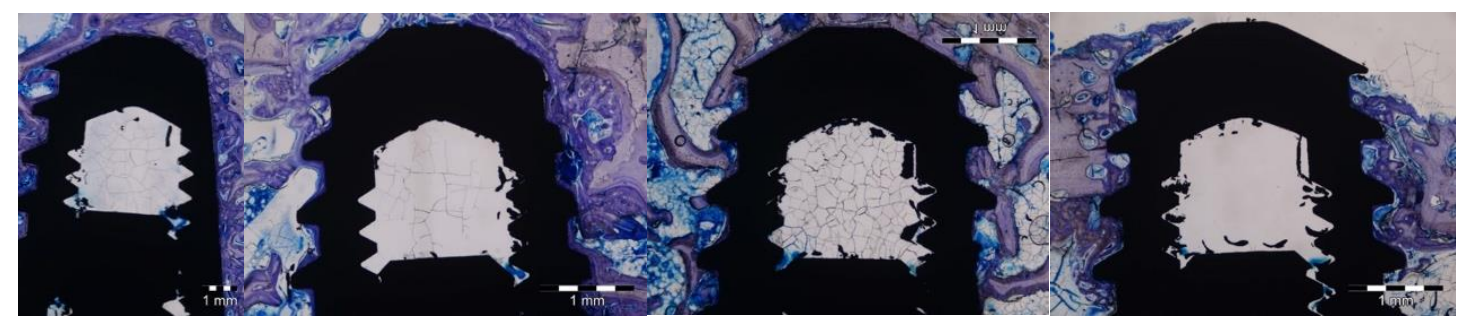

Figure 6. Histological section of implants. From left to right; empty control defect, defect filled with †autogenous graft, ${ }^{\star}$ xenograft, and ${ }^{\S}$ autogenous \& xenograft combination.

\subsection{Histomorphometric findings.}

\subsubsection{New Bone Formation (NBF) values}

The histomorphometric analysis of all the sections showed that the value of NBF was higher in vitamin D3-loaded groups than in the group without vitamin D3 $(P<0.001)$. The findings showed significant differences between the vitamin D3-loaded sections and the vitamin D3 not-loaded sections among the empty-grafted group, autografted group, xenografted group, and autografted xenografted group (Table 3 and 4).

Table 3. New bone formation (NBF) values between groups.

\begin{tabular}{|c|c|c|c|c|}
\hline \multirow{2}{*}{ Vitamin $\mathbf{D}_{3}$} & \multirow{2}{*}{ Time } & \multirow{2}{*}{ Group } & \multicolumn{2}{|c|}{ NBF\% } \\
\hline & & & Mean \pm SD & Min-Max \\
\hline \multirow{8}{*}{$\begin{array}{ll}\text { Vitamin } & D_{3} \\
(-) & \end{array}$} & \multirow[t]{4}{*}{3 Weeks } & Empty & $38.17 \pm 2.4$ & $35.8-40.6$ \\
\hline & & Autografted & $41.45 \pm 3.02$ & $38.25-44.26$ \\
\hline & & Xenografted & $39.86 \pm 2.8$ & $36.78-42.26$ \\
\hline & & Autografted and xenografted & $43.31 \pm 0.66$ & $42.68-44$ \\
\hline & \multirow[t]{4}{*}{6 Weeks } & Empty & $59.93 \pm 1.52$ & $58.26-61.24$ \\
\hline & & Autografted & $63.71 \pm 1.31$ & $62.36-64.98$ \\
\hline & & Xenografted & $61.87 \pm 1.41$ & $60.36-63.14$ \\
\hline & & Autografted and xenografted & $67.96 \pm 1.13$ & $66.87-69.12$ \\
\hline \multirow{8}{*}{$\begin{array}{ll}\text { Vitamin } & D_{3} \\
(+) & \end{array}$} & \multirow[t]{4}{*}{3 Weeks } & Empty & $44.31 \pm 2.45$ & $42.16-46.98$ \\
\hline & & Autografted & $47.6 \pm 1.86$ & $46.12-49.69$ \\
\hline & & Xenografted & $46.45 \pm 2.07$ & $44.93-48.81$ \\
\hline & & Autografted and xenografted & $49.63 \pm 1.53$ & $48.25-51.27$ \\
\hline & \multirow[t]{4}{*}{6 Weeks } & Empty & $66.75 \pm 0.56$ & $66.14-67.25$ \\
\hline & & Autografted & $69.55 \pm 0.53$ & $69.14-70.14$ \\
\hline & & Xenografted & $68.39 \pm 1.33$ & $67.14-69.78$ \\
\hline & & Autografted and xenografted & $74.25 \pm 0.96$ & $73.14-74.87$ \\
\hline
\end{tabular}


Table 4. Comparison between different sacrification times and groups on NBF values.

\begin{tabular}{c|c|c|c|c} 
Time & Group & & Difference & $\boldsymbol{P}$ \\
\hline \multirow{2}{*}{$\begin{array}{l}\text { Third } \\
\text { week }\end{array}$} & Empty & D vit (+) - D vit (-) & 6.140 & $0.002^{*}$ \\
\cline { 2 - 5 } & Autografted & D vit (+)- D vit (-) & 6.147 & $0.002^{*}$ \\
\cline { 2 - 5 } & Xenografted & D vit (+)- D vit (-) & 6.587 & $0.001^{*}$ \\
\cline { 2 - 5 } & Autografted and xenografted & D vit (+)- D vit (-) & 6.317 & $0.001^{*}$ \\
\hline \multirow{3}{*}{$\begin{array}{c}\text { Sixth } \\
\text { week }\end{array}$} & Empty & D vit (+)- D vit (-) & 6.823 & $<0.001^{*}$ \\
\cline { 2 - 5 } & Autografted & D vit (+)- D vit (-) & 5.840 & $<0.001^{*}$ \\
\cline { 2 - 5 } & Xenografted & D vit (+)- D vit (-) & 6.520 & $<0.001^{*}$ \\
\cline { 2 - 5 } & Autografted and xenografted & 6.290 & $<0.001^{*}$
\end{tabular}

${ }^{*} P<0.05$.

\subsubsection{Bone Implant Contact (BIC) values}

Similar to the findings of NBF, the histomorphometric analysis in all sections showed that the value of BIC was higher in the vitamin D3-loaded group than in the vitamin D3 notloaded group. In the group sacrificed after 3 weeks, the vitamin D3-loaded sections showed a statistically significant difference in the empty-grafted group, xenografted group, autografted and xenografted group $(P<0.001)$, and autografted group $(P=0.001)$ compared with the vitamin D3 not-loaded sections.

In the group sacrificed after 6 weeks, the vitamin D3-loaded sections showed a statistically significant difference in the empty-grafted group, autografted group, xenografted group, and autografted and xenografted group compared with the vitamin D3 not-loaded sections $(P<0.001)$ (Table 5, 6 and Figure 7).

Table 5. Mean values on BIC values between groups.

\begin{tabular}{|c|c|c|c|c|}
\hline \multirow{2}{*}{ Vitamin D3 } & \multirow{2}{*}{ Time } & \multirow{2}{*}{ Group } & \multicolumn{2}{|c|}{ BIC\% } \\
\hline & & & Mean \pm SD & Min-Max \\
\hline \multirow{8}{*}{ Vitamin $\mathbf{D}_{3}(-)$} & \multirow[t]{4}{*}{3 Weeks } & Empty & $40.81 \pm 0.96$ & $39.87-41.78$ \\
\hline & & Autografted & $46.64 \pm 1.62$ & $44.95-48.19$ \\
\hline & & Xenografted & $45.03 \pm 1.64$ & $43.67-46.85$ \\
\hline & & Autografted and xenografted & $49.55 \pm 0.68$ & $48.76-50.01$ \\
\hline & \multirow[t]{4}{*}{6 Weeks } & Empty & $62.76 \pm 1.42$ & $61.67-64.36$ \\
\hline & & Autografted & $68.27 \pm 1.04$ & $67.23-69.31$ \\
\hline & & Xenografted & $67.25 \pm 0.99$ & $66.26-68.24$ \\
\hline & & Autografted and xenografted & $70.45 \pm 0.8$ & $69.87-71.36$ \\
\hline \multirow[t]{8}{*}{ Vitamin $D_{3}(+)$} & \multirow[t]{4}{*}{3 Weeks } & Empty & $48.6 \pm 1.1$ & $47.89-49.87$ \\
\hline & & Autografted & $50.72 \pm 0.86$ & $50.18-51.71$ \\
\hline & & Xenografted & $49.51 \pm 0.45$ & $49.12-50.01$ \\
\hline & & Autografted and xenografted & $54.26 \pm 0.66$ & $53.79-55.01$ \\
\hline & \multirow[t]{4}{*}{6 Weeks } & Empty & $71.46 \pm 1.05$ & $70.28-72.26$ \\
\hline & & Autografted & $74.65 \pm 2.15$ & $72.25-76.39$ \\
\hline & & Xenografted & $73.19 \pm 2.7$ & $71.18-76.25$ \\
\hline & & Autografted and xenografted & $82.59 \pm 2.09$ & $80.25-84.28$ \\
\hline
\end{tabular}

Table 6. Comparison between different sacrifice times and groups on BIC values.

\begin{tabular}{l|l|l|c|c} 
Time & Group & & Difference & $\boldsymbol{P}$ \\
\hline \multirow{3}{*}{ 3 Weeks } & Empty & D vit (+)- D vit (-) & 7.787 & $<0.001^{*}$ \\
\cline { 2 - 4 } & Autografted & D vit (+)- D vit (-) & 4.077 & $0.001^{*}$ \\
\cline { 2 - 4 } & Xenografted & D vit (+)- D vit (-) & 4.487 & $<0.001^{*}$ \\
\cline { 2 - 4 } & Autografted and xenografted & D vit (+)- D vit (-) & 4.713 & $<0.001^{*}$ \\
\hline \multirow{6}{*}{6 Weeks } & Empty & D vit (+)- D vit (-) & 8.703 & $<0.001^{*}$ \\
\cline { 2 - 4 } & Autografted & D vit (+)-D vit (-) & 6.387 & $<0.001^{*}$ \\
\cline { 2 - 4 } & Xenografted & D vit (+)-D vit (-) & 5.933 & $<0.001^{*}$ \\
\cline { 2 - 4 } & Autografted and xenografted & D vit (+) - D vit (-) & 12.140 & $<01^{*}$
\end{tabular}

${ }^{*} P<0.05$. 


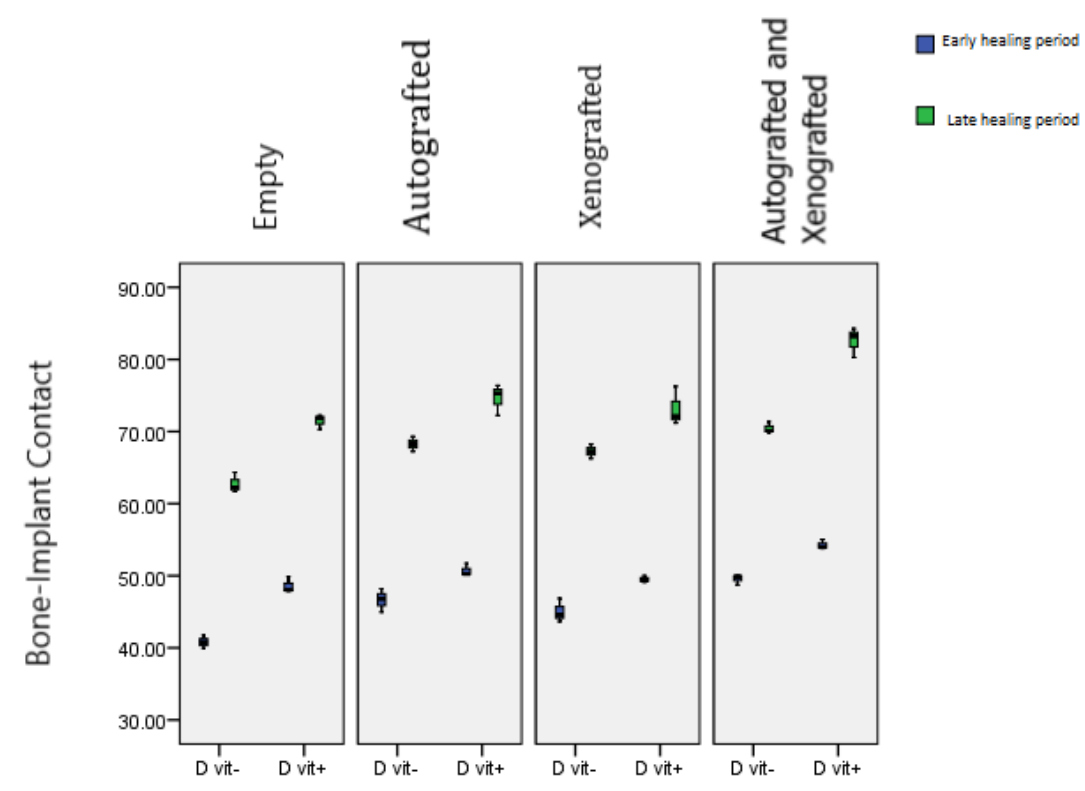

Figure 7. Box-whisker plot graphics of bone-implant contact values.

\subsection{Discussion.}

In dentistry, the main purpose of tissue engineering is to support the regeneration of alveolar bone affected by tooth extraction, trauma, or periodontal degeneration. Several treatment methods and novel regeneration materials are being investigated and explored by researchers and experts in the field. Autogenous grafts involving osteoinduction, osteoconduction, and osteogenesis properties continue to be prioritized among all graft materials. The mixing of xenografts and particulate autogenous grafts might enhance the capacity of osseointegration, but long-term studies are still needed.

During the regeneration process, both donor and donor sites have their own essential roles. The recipient area's regeneration capacity is associated with the general health of the patient. Systemic diseases, parafunction, and lack of nutritious and essential diet all affect the regeneration capacity. Essentials refer to the vitamins and essential oils in the body that work like catalyzers. In this respect, vitamin D3 is the most efficient catalyzer for hard tissues. Vitamin D3 levels directly affect mineral homeostasis, especially calcium and phosphate metabolism. These minerals, in return, affect the bone density and bone remodeling processes. This means that the presence of vitamin D3 is an indispensable component of bone regeneration.

This study compared the impact of continuous-effect vitamin D3 with different graft materials on bone regeneration of surgically created box-size defects. The continuous effect was provided by loaded PLA nanoparticles. Moreover, the healing periods of 3 weeks and 6 weeks are frequently selected in bone graft experiments in the sheep ilium [24]. The regeneration and osseointegration capacity at early stages could be enhanced with the sustained release of vitamin D3.

Lalloz et al. investigated the cholecalciferol's surface chemistry on the skin, and they found that PLA nanoparticles improved drug penetration [27]. PLA-based hydrogels use variable situations [28].

In addition, Zheng et al. used rifapentine PLA for drug release characteristics and tissue distribution. In the rabbit model [29], rifapentine was used in PLA sustained-release microspheres. The HPLC analysis maintained the level of rifapentine above the minimum 
inhibitory concentration. This study showed that PLA might be usable for experimental animal models.

Vitamin D3 plays an essential role in various systems in the body. Its effect on mineral homeostasis impacts the musculoskeletal systems, inflammatory response [17,29,30,31], and glucose metabolism [32], and can prevent types of cancers [33].

Vitamin D3 intake depends on dietary habits and exposure to ultraviolet (UV) lights. Nemeh et al. studied bone metabolism and calcium homeostasis parameters in goats and sheep [34]. The study showed that 13 weeks after the sacrifice, a vitamin D3 reduced diet and decreased UVB exposure group had lower bone mineral density, lower values of bone markers, growth hormone, and insulin-like growth factor in serum, and higher tachysterol and lower lumisterol content in the skin. In addition, Kohler et al. studied the influence of altitude on vitamin D3 and bone metabolism within the bone strength index values. In this study, the animals were separated to lowland and normal altitude. The findings showed that the animals in the lowland had lower values of vitamin D3 [35].

Some dental applications showed greater levels of vitamin D3 in the human body. Collins et al. performed a trial on cats. They were able to determine the rate and amount of orthodontic tooth treatment by injecting 1,25 dihydroxycholecalciferol $(1,25 \mathrm{D})$ into the periodontal ligament. After 21 days, the experimental tooth moved $60 \%$ further in the injected group compared with the control group [13]. Moreover, Kawakami et al. performed trial research on rats. In their study, they specifically investigated the effect of a local injection of 1,25-dihydroxyvitamin D3 on bone formation for tooth stabilization after experimental tooth movement in rats and the mineral apposition rate (MAR) value. A significant increase in the MAR value was found between the test and control groups [16]. These findings showed that vitamin D affects the apposition and repositioning of bone. Ciur et al. performed a similar study on humans considering the influence of local administration of vitamin D3 on the rate of orthodontic tooth movement. Using the split-mouth technique between intraligamentary administration of vitamin D3-applied group and the not-applied group, an average of 70\% more tooth movement was visible for the experimental teeth than the control ones [36].

$\mathrm{Wu}$ et al. further studied vitamin D3 and insulin combined treatment on titanium implant osseointegration among diabetes mellitus rats. The study exemplified that insulin and vitamin D3 combined treatment may effectively enhance implant fixation in diabetes mellitus [37]. Moreover, Dvorak et al. experimented with the impact of dietary vitamin D3 on osseointegration in ovariectomized rats. The study found that the vitamin D3 free group showed lower values than the standard dietary group on BIC and 25-hydroxyvitamin D serum levels [38]. For ethical reasons in our country (Turkey), the dietary restriction was forbidden on herbivore animals, and therefore any possible effect of dietary vitamin D3 deficiency could not be explored in this study.

Li et al. investigated the inhibitory effects of 25-hydroxyvitamin D3 [25(OH)D3)loaded PLA microspheres] on the inflammatory response on diabetic periodontitis in rat bone marrow stromal cells cultured with the Aggregatibacter actinomycetemcomitans model. They found that vitamin D3-loaded microspheres could ameliorate diabetic periodontitis by inhibiting inflammatory response [17]. Mangano et al. conducted a comprehensive retrospective study from January 2003 to December 2017 based on early dental implant failure (EDIF). In this study, 885 patients treated with 1740 fixtures were included. Within the study limitations, a dramatic increase in EDIFs was found when the vitamin D3 levels were lowered [39]. 
Zhou et al. performed a study to understand the effect of 1,25 dihydroxy vitamin D3 on titanium implant osseointegration in osteoporotic rats. After ovariectomy, the animals were separated into two groups; one group was administered $1,25(\mathrm{OH})_{2} \mathrm{D}_{3}$ through oral gavage at $0.1 \mathrm{~g} /(\mathrm{kg} \cdot$ day $)$, and the other group was normal. The test group showed greater values than the normal group [40]. Naito et al. studied the influence of 1 $\alpha .25$-dihydroxyvitamin D3 coating on implant osseointegration in the rabbit tibia. The study's major finding was that the vitamin D3-coated implants showed higher values of BIC and NBF [41]. Cho et al. studied the coated anodized titanium implants with PLGA in the rabbit model. The results showed that stimulation of bone formation might occur adjacent to the surface of implants inserted into bone [42]. On the contrary, the present study aimed to use vitamin D in PLA nanoparticles such as a bone graft material. Similar positive findings were observed via the use of vitamin D3 in PLA.

\section{Conclusions}

In the present study, PLA nanoparticles and EE were produced on vitamin D3. PLA nanoparticles were favorable carrier systems for sustained release of vitamin D3. Sustainedrelease of vitamin D3 loaded into the PLA system increased the NBF\% and BIC\% values in the early and late healing groups around dental implants with box-shaped iliac bone defects in sheep. These findings revealed that vitamin D affects osseointegration affirmatively, yet these findings must be supported by further clinical studies.

\section{Funding}

This research received no external funding.

\section{Acknowledgments}

This manuscript extracts the doctoral thesis of İbrahim Ozan Mutlu submitted to the Institute of Health Sciences of Istanbul University in 2021. Implants were provided by Zinedent, Straumann Group Certified Partner, Ankara. The authors would like to thank Prof. Dr. Erdal Cevher from Istanbul University Pharmacy Institute for counseling and guidance in pharmacokinetic operations; Dr. Öznur İnan from İstanbul Mehmet Akif Ersoy Experimental Research Development and Training Center for assistance in the surgical procedure; Ali Baykuş for the statistical counseling; and Hasan Ekeer from Erciyes University for coordination of the non decalcified histological bone sections.

\section{Conflicts of Interest}

The authors declare no conflict of interest.

\section{References}

1. Winkler, T.; Sass, F.A.; Duda, G.N.; Schmidt-Bleek, K. A review of biomaterials in bone defect healing, remaining shortcomings and future opportunities for bone tissue engineering: The unsolved challenge. Bone \& joint research 2018, 7, 232-243, https://doi.org/10.1302/2046-3758.73.Bjr-2017-0270.R1.

2. Fernandez de Grado, G.; Keller, L.; Idoux-Gillet, Y.; Wagner, Q.; Musset, A.M.; Benkirane-Jessel, N.; Bornert, F.; Offner, D. Bone substitutes: a review of their characteristics, clinical use, and perspectives for large bone defects management. J Tissue Eng 2018, 9, https://doi.org/10.1177/2041731418776819.

3. Girón, J.; Kerstner, E.; Medeiros, T.; Oliveira, L.; Machado, G.M.; Malfatti, C.F.; Pranke, P. Biomaterials for bone regeneration: an orthopedic and dentistry overview. Brazilian journal of medical and biological research $=$ Revista brasileira de pesquisas medicas e biologicas 2021, 54, https://doi.org/10.1590/1414$431 \mathrm{X} 2021 \mathrm{e} 11055$. 
4. Akay, A.S.; Arısan, V.; Cevher, E.; Sessevmez, M.; Cam, B. Oxytocin-loaded sustained-release hydrogel graft provides accelerated bone formation: An experimental rat study. Journal of orthopaedic research : official publication of the Orthopaedic Research Society 2020, 38, 1676-1687, https://doi.org/10.1002/jor.24607.

5. Aral, A.; Yalçin, S.; Karabuda, Z.C.; Anil, A.; Jansen, J.A.; Mutlu, Z. Injectable calcium phosphate cement as a graft material for maxillary sinus augmentation: an experimental pilot study. Clinical oral implants research 2008, 19, 612-617, https://doi.org/10.1111/j.1600-0501.2007.01518.x.

6. Sheikh, Z.; Hamdan, N.; Ikeda, Y.; Grynpas, M.; Ganss, B.; Glogauer, M. Natural graft tissues and synthetic biomaterials for periodontal and alveolar bone reconstructive applications: a review. Biomaterials research 2017, 21, 1-20, https://doi.org/10.1186/s40824-017-0095-5.

7. Fukuba, S.; Okada, M.; Nohara, K.; Iwata, T. Alloplastic Bone Substitutes for Periodontal and Bone Regeneration in Dentistry: Current Status and Prospects. Materials (Basel, Switzerland) 2021, 14, https://doi.org/10.3390/ma14051096.

8. Correia, F.; Pozza, D.H.; Gouveia, S.; Felino, A.C.; Faria-Almeida, R. Advantages of Porcine Xenograft over Autograft in Sinus Lift: A Randomised Clinical Trial. Materials (Basel, Switzerland) 2021, 14, https://doi.org/10.3390/ma14123439.

9. Aludden, H.C.; Mordenfeld, A.; Hallman, M.; Dahlin, C.; Jensen, T. Lateral ridge augmentation with BioOss alone or Bio-Oss mixed with particulate autogenous bone graft: a systematic review. International journal of oral and maxillofacial surgery 2017, 46, 1030-1038, https://doi.org/10.1016/j.ijom.2017.03.008.

10. Zhao, R.; Yang, R.; Cooper, P.R.; Khurshid, Z.; Shavandi, A.; Ratnayake, J. Bone Grafts and Substitutes in Dentistry: A Review of Current Trends and Developments. Molecules 2021, 26, https://doi.org/10.3390/molecules26103007.

11. Christakos, S.; Dhawan, P.; Verstuyf, A.; Verlinden, L.; Carmeliet, G. Vitamin D: Metabolism, Molecular Mechanism of Action, and Pleiotropic Effects. Physiological reviews 2016, 96, 365-408, https://doi.org/10.1152/physrev.00014.2015.

12. Jarusriwanna, A.; Phusunti, S.; Chotiyarnwong, P.; Unnanuntana, A. High-dose versus low-dose ergocalciferol for correcting hypovitaminosis D after fragility hip fracture: a randomized controlled trial. BMC geriatrics 2021, 21, https://doi.org/10.1186/s12877-021-02023-1.

13. Collins, M.K.; Sinclair, P.M. The local use of vitamin D to increase the rate of orthodontic tooth movement. American journal of orthodontics and dentofacial orthopedics : official publication of the American Association of Orthodontists, its constituent societies, and the American Board of Orthodontics 1988, 94, 278-284, https://doi.org/10.1016/0889-5406(88)90052-2.

14. Maier, G.S.; Weissenberger, M.; Rudert, M.; Roth, K.E.; Horas, K. The role of vitamin D and vitamin D deficiency in orthopaedics and traumatology-a narrative overview of the literature. Annals of translational medicine 2021, 9, http://doi.org/10.21037/atm-21-779.

15. Cianciolo, G.; Cappuccilli, M.; Tondolo, F.; Gasperoni, L.; Zappulo, F.; Barbuto, S.; Iacovella, F.; Conte, D.; Capelli, I.; La Manna, G. Vitamin D Effects on Bone Homeostasis and Cardiovascular System in Patients with Chronic Kidney Disease and Renal Transplant Recipients. Nutrients 2021, 13, https://doi.org/10.3390/nu13051453.

16. Kawakami, M.; Takano-Yamamoto, T. Local injection of 1,25-dihydroxyvitamin D3 enhanced bone formation for tooth stabilization after experimental tooth movement in rats. Journal of bone and mineral metabolism 2004, 22, 541-546, https://doi.org/10.1007/s00774-004-0521-3.

17. Li, H.; Li, B.; Wang, Q.; Xiao, Y.; Chen, X.M.; Li, W. Attenuation of inflammatory response by $25-$ hydroxyvitamin D3-loaded polylactic acid microspheres in treatment of periodontitis in diabetic rats. The Chinese journal of dental research: the official journal of the Scientific Section of the Chinese Stomatological Association (CSA) 2014, 17, 91-98.

18. McCullough, P.J.; McCullough, W.P.; Lehrer, D.; Travers, J.B.; Repas, S.J. Oral and Topical Vitamin D, Sunshine, and UVB Phototherapy Safely Control Psoriasis in Patients with Normal Pretreatment Serum 25Hydroxyvitamin D Concentrations: A Literature Review and Discussion of Health Implications. Nutrients 2021, 13, https://doi.org/10.3390/nu13051511.

19. Kulda, V. [Vitamin D metabolism]. Vnitrni lekarstvi 2012, 58, 400-404.

20. Stalgis-Bilinski, K.L.; Boyages, J.; Salisbury, E.L.; Dunstan, C.R.; Henderson, S.I.; Talbot, P.L. Burning daylight: balancing vitamin D requirements with sensible sun exposure. Medical Journal of Australia 2011, 194, 345-348, https://doi.org/10.5694/j.1326-5377.2011.tb03003.x.

21. Holick, M.F. The vitamin D deficiency pandemic: Approaches for diagnosis, treatment and prevention. Reviews in endocrine \& metabolic disorders 2017, 18, 153-165, https://doi.org/10.1007/s11154-017-9424-1.

22. Li, G.; Zhao, M.; Xu, F.; Yang, B.; Li, X.; Meng, X.; Teng, L.; Sun, F.; Li, Y. Synthesis and Biological Application of Polylactic Acid. Molecules (Basel, Switzerland) 2020, 25, 5023, https://doi.org/10.3390/molecules25215023.

23. Balla, E.; Daniilidis, V.; Karlioti, G.; Kalamas, T.; Stefanidou, M.; Bikiaris, N.D.; Vlachopoulos, A.; Koumentakou, I.; Bikiaris, D.N. Poly(lactic Acid): A Versatile Biobased Polymer for the Future with Multifunctional Properties-From Monomer Synthesis, Polymerization Techniques and Molecular Weight Increase to PLA Applications. Polymers 2021, 13, https://doi.org/10.3390/polym13111822. 
24. Cakir, S.; Gultekin, B.A.; Karabagli, M.; Yilmaz, T.E.; Cakir, E.; Guzel, E.E.; Yalcin, S.; Mortellaro, C.; Mijiritsky, E. Histological Evaluation of the Effects of Growth Factors in a Fibrin Network on Bone Regeneration. The Journal of craniofacial surgery 2019, 30, 1078-1084, https://doi.org/10.1097/scs.0000000000005339.

25. Vora, L.K.; Donnelly, R.F.; Larrañeta, E.; González-Vázquez, P.; Thakur, R.R.S.; Vavia, P.R. Novel bilayer dissolving microneedle arrays with concentrated PLGA nano-microparticles for targeted intradermal delivery: Proof of concept. Journal of controlled release : official journal of the Controlled Release Society 2017, 265, 93-101, https://doi.org/10.1016/j.jconrel.2017.10.005.

26. Nakyinsige, K.; Che Man, Y.B.; Aghwan, Z.A.; Zulkifli, I.; Goh, Y.M.; Abu Bakar, F.; Al-Kahtani, H.A.; Sazili, A.Q. Stunning and animal welfare from Islamic and scientific perspectives. Meat Science 2013, 95, 352-361, https://doi.org/10.1016/j.meatsci.2013.04.006.

27. Lalloz, A.; Bolzinger, M.A.; Faivre, J.; Latreille, P.L.; Garcia Ac, A.; Rakotovao, C.; Rabanel, J.M.; Hildgen, P.; Banquy, X.; Briançon, S. Effect of surface chemistry of polymeric nanoparticles on cutaneous penetration of cholecalciferol. Int J Pharm 2018, 553, 120-131, https://doi.org/10.1016/j.ijpharm.2018.09.046.

28. Basu, A.; Kunduru, K.R.; Doppalapudi, S.; Domb, A.J.; Khan, W. Poly(lactic acid) based hydrogels. Adv Drug Deliv Rev 2016, 107, 192-205, https://doi.org/10.1016/j.addr.2016.07.004.

29. Zhang, Z.; Wu, L.; Li, H.; Long, Z.; Song, X. Drug Release Characteristics and Tissue Distribution of Rifapentine Polylactic Acid Sustained-Release Microspheres in Rabbits after Paravertebral Implantation. Iranian Red Crescent medical journal 2016, 18.

30. Ilie, P.C.; Stefanescu, S.; Smith, L. The role of vitamin D in the prevention of coronavirus disease 2019 infection and mortality. Aging Clin Exp Res 2020, 32, 1195-1198, https://dx.doi.org/10.1007\%2Fs40520020-01570-8.

31. Almeida Moreira Leal, L.K.; Lima, L.A.; Alexandre de Aquino, P.E.; Costa de Sousa, J.A.; Jataí Gadelha, C.V.; Felício Calou, I.B.; Pereira Lopes, M.J.; Viana Lima, F.A.; Tavares Neves, K.R.; Matos de Andrade, G.; Socorro de Barros Viana, G. Vitamin D (VD3) antioxidative and anti-inflammatory activities: Peripheral and central effects. Eur J Pharmacol 2020, 879, https://doi.org/10.1016/j.ejphar.2020.173099.

32. Garbossa, S.G.; Folli, F. Vitamin D, sub-inflammation and insulin resistance. A window on a potential role for the interaction between bone and glucose metabolism. Reviews in endocrine \& metabolic disorders 2017 , 18, 243-258, https://doi.org/10.1007/s11154-017-9423-2.

33. Garland, C.F.; Garland, F.C.; Gorham, E.D.; Lipkin, M.; Newmark, H.; Mohr, S.B.; Holick, M.F. The Role of Vitamin D in Cancer Prevention. American Journal of Public Health 2006, 96, 252-261, https://doi.org/10.2105/AJPH.2004.045260.

34. Nemeth, M.V.; Wilkens, M.R.; Liesegang, A. Vitamin D status in growing dairy goats and sheep: Influence of ultraviolet B radiation on bone metabolism and calcium homeostasis. Journal of dairy science 2017, 100, 8072-8086, https://doi.org/10.3168/jds.2017-13061.

35. Kohler, M.; Leiber, F.; Willems, H.; Merbold, L.; Liesegang, A. Influence of altitude on vitamin D and bone metabolism of lactating sheep and goats. J Anim Sci 2013, 91, 5259-5268, https://doi.org/10.2527/jas.20136702 .

36. Iosub Ciur, M.D.; Zetu, I.N.; Haba, D.; Viennot, S.; Bourgeois, D.; Andrian, S. Evaluation of the Influence of Local Administration of Vitamin D on the Rate of Orthodontic Tooth Movement. Revista medicochirurgicala a Societatii de Medici si Naturalisti din Iasi 2016, 120, 694-699.

37. Wu, Y.Y.; Yu, T.; Yang, X.Y.; Li, F.; Ma, L.; Yang, Y.; Liu, X.G.; Wang, Y.Y.; Gong, P. Vitamin D3 and insulin combined treatment promotes titanium implant osseointegration in diabetes mellitus rats. Bone 2013, 52, 1-8, https://doi.org/10.1016/j.bone.2012.09.005.

38. Dvorak, G.; Fügl, A.; Watzek, G.; Tangl, S.; Pokorny, P.; Gruber, R. Impact of dietary vitamin D on osseointegration in the ovariectomized rat. Clinical oral implants research 2012, 23, 1308-1313, https://doi.org/10.1111/j.1600-0501.2011.02346.x.

39. Guido Mangano, F.; Ghertasi Oskouei, S.; Paz, A.; Mangano, N.; Mangano, C. Low serum vitamin D and early dental implant failure: Is there a connection? A retrospective clinical study on 1740 implants placed in 885 patients. Journal of dental research, dental clinics, dental prospects 2018, 12, 174-182, https://doi.org/10.15171/joddd.2018.027.

40. Zhou, C.; Li, Y.; Wang, X.; Shui, X.; Hu, J. 1,25Dihydroxy vitamin D(3) improves titanium implant osseointegration in osteoporotic rats. Oral surgery, oral medicine, oral pathology and oral radiology 2012, 114, S174-178, https://doi.org/10.1016/j.oooo.2011.09.030.

41. Naito, Y.; Jimbo, R.; Bryington, M.S.; Vandeweghe, S.; Chrcanovic, B.R.; Tovar, N.; Ichikawa, T.; Paulo G, C.; Wennerberg, A. The influence of 1 $\alpha .25$-dihydroxyvitamin $\mathrm{d} 3$ coating on implant osseointegration in the rabbit tibia. J Oral Maxillofac Res 2014, 5, https://doi.org/10.5037/jomr.2014.5303.

42. Cho, Y.J.; Heo, S.J.; Koak, J.Y.; Kim, S.K.; Lee, S.J.; Lee, J.H. Promotion of osseointegration of anodized titanium implants with a 1 $\alpha, 25$-dihydroxyvitamin D3 submicron particle coating. The International journal of oral \& maxillofacial implants 2011, 26, 1225-1232. 